\title{
$n=0$ Energy Level Present in the Hydrogen Atom
}

\author{
Koshun Suto ${ }^{1}$ \\ ${ }^{1}$ Chudaiji Buddhist Temple, Isesaki, Japan \\ Correspondence: Koshun Suto, Chudaiji Buddhist Temple, 5-24, Oote-Town, Isesaki, 372-0048, Japan. \\ Tel: 81-270-23-9980. E-mail: koshun_suto129@mbr.nifty.com
}

Received: September 10, 2014 Accepted: September 17, $2014 \quad$ Online Published: September 20, 2014

doi:10.5539/apr.v6n5p109

URL: http://dx.doi.org/10.5539/apr.v6n5p109

\begin{abstract}
In this paper an equation with higher precision than the equation of quantum mechanics is derived as an equation describing the energy levels of the hydrogen atom using only the principal quantum number $n$. That equation predicts the existence of an $n=0$ energy level. However, the state where $n=0$ is not an energy level of the electron comprising the hydrogen atom. The electron in this state forms a pair with a positron, which is the electron's antiparticle. The relativistic energy of the individual particles forming the pair is zero, but their momentum is not zero. The momentums of these particles are equal in magnitude but opposite in direction. By forming a pair, the sum of the momentums of two types of particles becomes zero. It is very significant that even the energy level of the electron pairs which comprise the vacuum have been incorporated into the formula for the energy of the hydrogen atom.
\end{abstract}

Keywords: hydrogen atom, energy level, Einstein's energy-momentum relationship, Dirac relativistic wave equation

\section{Introduction}

One of the most important relationship in the special theory of relativity is as follows:

$$
\left(m c^{2}\right)^{2}=\boldsymbol{p}^{2} c^{2}+\left(m_{0} c^{2}\right)^{2}
$$

Here, $m c^{2}$ is the relativistic energy of an object or a particle.

Einstein's relationship (1) is used to describe the energy and momentum in free space. The rest mass energy of an object with a rest mass of $m_{0}$ is given by the following famous equation:

$$
E=m_{0} c^{2}
$$

Here, the author will consider the case where an electron in free space forms a hydrogen atom due to the attraction of the atomic nucleus (i.e., the proton).

According to the famous virial theorem, if $K$ is taken to be the kinetic energy of the entire system, and $V$ is taken to be the potential energy of the entire system, then the following relation holds between $K$ and $V$ :

$$
\langle K\rangle=-\frac{1}{2}\langle V\rangle .
$$

The average time of $K$ is equal to $-1 / 2$ the time average of $V$. Also, the sum of the time average $K$ of the kinetic energy of the entire system and the time average of the total mechanical energy $E$ of the entire system becomes 0 . That is,

$$
\langle K\rangle+\langle E\rangle=0
$$

Next, if Equations (3) and (4) are combined, the result is as follows:

$$
-\langle E\rangle=\langle K\rangle=-\frac{1}{2}\langle V\rangle .
$$

In the entire system, $1 / 2$ of the reduced potential energy is converted into kinetic energy of the entire system, and the remaining energy is emitted to the outside of the atom as a photon. 
In the hydrogen atom, the atomic nucleus can be regarded as being heavy and stationary, and thus the discussion here will take the kinetic energy and potential energy of the entire system to be the energy of the electron.

Now, consider the case where an electron stationary in free space emits a photon without absorbing energy from the outside, and at the same time acquires the same amount of kinetic energy as the energy of the photon. In this situation, there must be an energy source for the kinetic energy and photon in order to satisfy the law of conservation of energy. If the virial theorem is taken into account, then potential energy is the energy source. However, the energy possessed by the electron when it is stationary is only its rest mass energy.

Thus, the author presented the following equation as an equation indicating the relationship between the rest mass energy and potential energy of the electron (Suto, 2009).

$$
V(r)=-\Delta m_{\mathrm{e}} c^{2} .
$$

According to this equation, the potential energy of the hydrogen atom corresponds to the reduction in rest mass energy of the electron in the atom.

This shows that the following inequalities hold for the total mechanical energy and potential energy of the electron in the hydrogen atom.

$$
\begin{aligned}
& -\frac{1}{2} m_{\mathrm{e}} c^{2} \leq E<0 . \\
& -m_{\mathrm{e}} c^{2} \leq V(r)<0 .
\end{aligned}
$$

From these equations, it can be predicted, even without using quantum mechanics, that there is a lower limit to the energy values of the hydrogen atom.

Incidentally, in the classical quantum theory of Bohr, the energy levels of the hydrogen atom can be expressed with the following equation:

$$
E_{\mathrm{B}, n}=-\frac{1}{2}\left(\frac{1}{4 \pi \varepsilon_{0}}\right)^{2} \frac{m_{\mathrm{e}} e^{4}}{\hbar^{2}} \cdot \frac{1}{n^{2}}=-\frac{\alpha^{2} m_{\mathrm{e}} c^{2}}{2 n^{2}}, \quad n=1,2, \cdots
$$

Here, the B in $E_{\mathrm{B}}$ indicates the equation derived by Bohr.

In the theory of Dirac, the energy levels of the hydrogen atom can be expressed with the following equation (Schiff, 1968):

$$
E_{n}=m_{\mathrm{e}} c^{2}\left[1-\frac{\alpha^{2}}{2 n^{2}}-\frac{\alpha^{4}}{2 n^{4}}\left(\frac{n}{|k|}-\frac{3}{4}\right)\right] .
$$

When $n /|k|=1$ in Equation (10), Equation (10) becomes as follows.

$$
E_{n}=m_{\mathrm{e}} c^{2}\left(1-\frac{\alpha^{2}}{2 n^{2}}-\frac{\alpha^{4}}{8 n^{4}}\right) .
$$

$E_{n}$ of Equations (10) and (11) define an absolute quantity, which includes the electron's rest mass energy. Whereas $E_{\mathrm{B}, n}$ in Equation (9) expresses the reduction in rest mass energy of the electron. Thus, if Equation (11) is written from the same standpoint as Equation (9), the result is as follows:

$$
E_{\mathrm{D}, n}=-\frac{\alpha^{2} m_{\mathrm{e}} c^{2}}{2 n^{2}}\left(1+\frac{\alpha^{2}}{4 n^{2}}\right)
$$

Here, the $\mathrm{D}$ in $E_{\mathrm{D}}$ indicates the equation derived by Dirac.

Incidentally, the lower limit of $E_{n}$ predicted by quantum mechanics is roughly $-\alpha^{2} m_{\mathrm{e}} c^{2} / 2$, but classically values up to $-m_{\mathrm{e}} c^{2} / 2$ are possible. The difference between these energies is unnaturally large. Thus, the following section of this paper examines whether an energy level lower than the value predicted by quantum mechanics actually exists. 


\section{Theoretical Investigation}

Einstein's relation between energy and momentum (1) was presented in the introduction. However, since Equation (1) does not include the potential energy of the electron, this equation cannot be applied to the electron in the hydrogen atom. Even so, the author has derived the following relationship for the bound electron in a hydrogen atom, which must take into account the Coulomb potential (Suto, 2011):

$$
E_{\mathrm{re}, n}^{2}+\boldsymbol{p}_{n}^{2} c^{2}=\left(m_{\mathrm{e}} c^{2}\right)^{2}
$$

Also, $E_{\mathrm{re}, h}$ has been defined as follows.

$$
E_{\mathrm{re}, n}=m_{\mathrm{e}} c^{2}+E_{n}, \quad n=1,2, \cdots
$$

Here, $E_{\mathrm{re}, n}$ is the relativistic energy of the electron, and the electron's energy is described on an absolute scale. Incidentally, the following equation was derived from Einstein's relationship (1).

$$
E=\frac{m_{0} c^{2}}{\left(1-v^{2} / c^{2}\right)^{1 / 2}} .
$$

When the same logic is applied to Equation (13), the following equation can be derived.

$$
E_{\mathrm{re}}=\frac{m_{\mathrm{e}} c^{2}}{\left(1+v^{2} / c^{2}\right)^{1 / 2}} .
$$

This equation can be written as follows:

$$
E_{\mathrm{re}}=\frac{m_{\mathrm{e}} c^{2}\left(1-v^{2} / c^{2}\right)^{1 / 2}}{\left(1+v^{2} / c^{2}\right)^{1 / 2}\left(1-v^{2} / c^{2}\right)^{1 / 2}}=\left(1-\frac{v^{4}}{c^{4}}\right)^{-1 / 2} m_{\mathrm{e}} c^{2}\left(1-\frac{v^{2}}{c^{2}}\right)^{1 / 2} .
$$

If part of this equation is replaced with its Taylor expansion, the result is as follows:

$$
m_{\mathrm{e}} c^{2}\left(1-\frac{v^{2}}{c^{2}}\right)^{1 / 2}=m_{\mathrm{e}} c^{2}\left(1-\frac{v^{2}}{2 c^{2}}-\frac{v^{4}}{8 c^{4}}+\cdots\right) .
$$

As a result, it is possible to write Equation (17) as follows:

$$
E_{\mathrm{re}}=\left(1-\frac{v^{4}}{c^{4}}\right)^{-1 / 2} m_{\mathrm{e}} c^{2}\left(1-\frac{v^{2}}{2 c^{2}}-\frac{v^{4}}{8 c^{4}}+\cdots\right) .
$$

In classical terms, the velocity of the electron in the hydrogen atom is about $\alpha c / n$, and thus the following relation holds.

$$
\left(1-\frac{v^{4}}{c^{4}}\right)^{-1 / 2} \approx 1
$$

Incidentally, Equation (19) is similar to the Taylor expansion of the following equation.

$$
E_{n}=m_{\mathrm{e}} c^{2}\left(1-\frac{\alpha^{2}}{n^{2}}\right)^{1 / 2}=m_{\mathrm{e}} c^{2}\left(1-\frac{\alpha^{2}}{2 n^{2}}-\frac{\alpha^{4}}{8 n^{4}}+\cdots\right) .
$$

Thus this paper makes the following assumption, based on a comparison of Equation (19) and Equation (21).

$$
\frac{v}{c}=\frac{\alpha}{n}
$$

Here, $\alpha$ is the following fine structure constant. 


$$
\alpha=\frac{e^{2}}{4 \pi \varepsilon_{0} \hbar c}=7.2973525698 \times 10^{-3} .
$$

If Equation (22) is assumed, then Equation (19) can be written as follows.

$$
E_{\mathrm{re}, n}=\left(1-\frac{\alpha^{4}}{n^{4}}\right)^{-1 / 2} m_{\mathrm{e}} c^{2}\left(1-\frac{\alpha^{2}}{2 n^{2}}-\frac{\alpha^{4}}{8 n^{4}}+\cdots\right) .
$$

Here $\left(1-\alpha^{4} / n^{4}\right)^{-1 / 2} \approx 1$, and thus if $E_{\mathrm{re}, n}$ is written from the same standpoint as $E_{\mathrm{D}}$,

$$
E_{n} \approx-\frac{\alpha^{2} m_{\mathrm{e}} c^{2}}{2 n^{2}}\left(1+\frac{\alpha^{2}}{4 n^{2}}-\cdots\right)=E_{\mathrm{D}, n} .
$$

This matches the Dirac equation (12) (The 3th term exists even in the Dirac equation, but it has simply been omitted). Also, if the 2nd and subsequent terms in parentheses in Equation (25) are discarded,

$$
E_{n} \approx-\frac{\alpha^{2} m_{\mathrm{e}} c^{2}}{2 n^{2}}=E_{\mathrm{B}, n}
$$

Then this matches the Bohr equation (9).

Incidentally, if Equation (21) is taken into account, the Dirac equation (12) is equivalent to the following equation.

$$
E_{\mathrm{D}, n}=m_{\mathrm{e}} c^{2}\left[\left(1-\frac{\alpha^{2}}{n^{2}}\right)^{1 / 2}-1\right] .
$$

However, this equation cannot be derived at the level of classical theory. However, the following equation can be derived from Equation (24).

$$
E_{n}=m_{\mathrm{e}} c^{2}\left[\left(1-\frac{\alpha^{4}}{n^{4}}\right)^{-1 / 2}\left(1-\frac{\alpha^{2}}{n^{2}}\right)^{1 / 2}-1\right] .
$$

Thus, as an equation including only the quantum number $n$, this paper predicts that Equation (28) will have higher precision than Equation (27). In addition, Equation (28) can also be inferred from Equation (16), and this is equivalent to the following equation.

$$
\begin{aligned}
E_{n}=E_{\mathrm{re}, n}-m_{\mathrm{e}} c^{2} & =m_{\mathrm{e}} c^{2}\left[\frac{1}{\left(1+\alpha^{2} / n^{2}\right)^{1 / 2}}-1\right] \\
& =m_{\mathrm{e}} c^{2}\left[\left(\frac{n^{2}}{n^{2}+\alpha^{2}}\right)^{1 / 2}-1\right] .
\end{aligned}
$$

Here, if the energy when $n=1$ is derived from Equations (26), (25) and (29), the result is as follows:

$$
\begin{aligned}
& E_{\mathrm{B}, 1}=-13.605 \underline{69} \mathrm{eV} . \\
& E_{\mathrm{D}, 1}=-13.605149 \underline{19} \mathrm{eV} . \\
& E_{1}=-13.605149 \underline{21} \mathrm{eV} .
\end{aligned}
$$

The values of $E_{\mathrm{D}, 1}$ and $E_{1}$ are extremely close, and thus it is difficult to determine which of the two that the observed values match. However, in terms of suggesting the existence of $E_{0}$, this paper predicts that Equation (29) will have greater precision than Equation (27). That is, 


$$
E_{0}=-m_{\mathrm{e}} c^{2}=-0.510998928 \mathrm{MeV} .
$$

Also, if this energy is written using $E_{\mathrm{re}}$ defined in Equation (14),

$$
E_{\mathrm{re}, 0}=0 .
$$

From this it is evident that the mass of the electron in this state is zero.

In the end, when Equation (29.2) is given up to the quantum condition, the result is as follows.

$$
E_{n}=m_{\mathrm{e}} c^{2}\left[\left(\frac{n^{2}}{n^{2}+\alpha^{2}}\right)^{1 / 2}-1\right], \quad n=0,1,2, \cdots .
$$

In quantum mechanics, topics such as the existence of an $n=0$ energy level were not discussed. However the next section of this paper will examine an $n=0$ state.

\section{Possible Existence of an $\boldsymbol{n}=\mathbf{0}$ Energy Level}

Assuming that an $n=0$ energy level can exist, let us calculate the distance $r$ between the electron and the atomic nucleus.

First, the energy of the hydrogen atom is given by the following equation.

$$
E_{n}=-\frac{1}{2} \frac{1}{4 \pi \varepsilon_{0}} \frac{e^{2}}{r_{n}} .
$$

Due to this equation and Equation (14), the relativistic energy $E_{\mathrm{re}, n}$ becomes as follows.

$$
E_{\mathrm{re}, n}=m_{\mathrm{e}} c^{2}-\frac{1}{2} \frac{1}{4 \pi \varepsilon_{0}} \frac{e^{2}}{r_{n}} .
$$

In addition, if Equation (29.1) is used for reference, the following equation holds.

$$
m_{\mathrm{e}} c^{2}-\frac{1}{2} \frac{1}{4 \pi \varepsilon_{0}} \frac{e^{2}}{r_{n}}=\frac{m_{\mathrm{e}} c^{2}}{\left(1+\alpha^{2} / n^{2}\right)^{1 / 2}} .
$$

If $r_{n}$ is found from that,

$$
r_{n}=\frac{1}{2} \frac{e^{2}}{4 \pi \varepsilon_{0} m_{\mathrm{e}} c^{2}}\left[1-\frac{1}{\left(1+\alpha^{2} / n^{2}\right)^{1 / 2}}\right]^{-1}=\frac{r_{\mathrm{e}}}{2} \frac{\left(n^{2}+\alpha^{2}\right)^{1 / 2}}{\left(n^{2}+\alpha^{2}\right)^{1 / 2}-n} .
$$

Here, when $n=0$,

$$
r_{0}=\frac{r_{\mathrm{e}}}{2} .
$$

Since the radius of the atomic nucleus of the hydrogen atom (i.e., the proton) is about $r_{\mathrm{e}} / 4$, then from the standpoint of distance, it is possible for an electron to exist at an $n=0$ energy level.

However, the next step will be to confirm that the $r_{0}$ in Equation (38) is a meaningful physical quantity when discussed within the scope of classical quantum theory (i.e., that $r$ has meaning as an expected value in quantum mechanics).

Next, let's try transforming Equation (37).

$$
r_{n}=\frac{r_{\mathrm{e}}}{2} \cdot \frac{\left(n^{2}+\alpha^{2}\right)^{1 / 2}\left[\left(n^{2}+\alpha^{2}\right)^{1 / 2}+n\right]}{\left[\left(n^{2}+\alpha^{2}\right)^{1 / 2}-n\right]\left[\left(n^{2}+\alpha^{2}\right)^{1 / 2}+n\right]}=\frac{r_{\mathrm{e}}}{2} \cdot \frac{\left(n^{2}+\alpha^{2}\right)+n^{2}\left(1+\alpha^{2} / n^{2}\right)^{1 / 2}}{\alpha^{2}} .
$$

In this way, 


$$
r_{n}=\frac{r_{\mathrm{e}}}{2}+\frac{n^{2} r_{\mathrm{e}}}{2 \alpha^{2}}+\frac{n^{2} r_{\mathrm{e}}\left(1+\alpha^{2} / n^{2}\right)^{1 / 2}}{2 \alpha^{2}}
$$

In Equation (40),

$$
n \rightarrow \infty, \quad\left(1+\frac{\alpha^{2}}{n^{2}}\right)^{1 / 2} \rightarrow 1 .
$$

Thus, Equation (40) converges on the following equation.

$$
r_{n} \approx \frac{r_{\mathrm{e}}}{2}+\frac{n^{2} r_{\mathrm{e}}}{\alpha^{2}}=\frac{r_{\mathrm{e}}}{2}+n^{2} a_{\mathrm{B}}, \quad n=0,1,2, \cdots
$$

Incidentally, the electron orbital radius $r_{n}$ of Bohr, derived from classical quantum theory, is given by the following equation.

$$
r_{n}=n^{2} a_{\mathrm{B}}, \quad n=1,2, \cdots
$$

If Equation (42) and Equation (43) are compared, in Equation (42) $n$ starts from 0, and an $r_{\mathrm{e}} / 2$ term is newly added.

When Bohr found Equation (9), he first assumed the quantum condition $p_{n} \cdot 2 \pi r_{n}=2 \pi n \hbar$. However, in Equation (26) of this paper, it was determined that the Bohr equation is an approximation. In that case, doubts arise regarding the validity of Bohr's quantum condition.

Therefore, this paper carries out the actual derivation without assuming Bohr's quantum condition from the beginning.

First, from Equation (29.2),

$$
E_{\mathrm{re}, n}=m_{\mathrm{e}} c^{2}\left(\frac{n^{2}}{n^{2}+\alpha^{2}}\right)^{1 / 2} .
$$

If this equation is substituted into Equation (13), then $p_{n}$ is given by the following equation.

$$
p_{n}=m_{\mathrm{e}} c\left(\frac{\alpha^{2}}{n^{2}+\alpha^{2}}\right)^{1 / 2} .
$$

Thus, if Equations (45) and (39) are used, then $p_{n} \cdot 2 \pi r_{n}$ becomes as follows.

$$
\begin{aligned}
p_{n} \cdot 2 \pi r_{n} & =m_{\mathrm{e}} c\left(\frac{\alpha^{2}}{n^{2}+\alpha^{2}}\right)^{1 / 2} \cdot 2 \pi \cdot \frac{r_{\mathrm{e}}}{2} \frac{\left(n^{2}+\alpha^{2}\right)+n^{2}\left(1+\alpha^{2} / n^{2}\right)^{1 / 2}}{\alpha^{2}} \\
& =2 \pi m_{\mathrm{e}} c \cdot \frac{r_{\mathrm{e}}}{\alpha}\left(\frac{1}{n^{2}+\alpha^{2}}\right)^{1 / 2} \cdot \frac{1}{2}\left[\left(n^{2}+\alpha^{2}\right)+n\left(n^{2}+\alpha^{2}\right)^{1 / 2}\right] \\
& =2 \pi \hbar \cdot \frac{n}{2}\left[1+\left(1+\alpha^{2} / n^{2}\right)^{1 / 2}\right] \approx 2 \pi n \hbar .
\end{aligned}
$$

Due to the above considerations, it was determined that the quantum condition of Bohr is a condition which holds approximately.

\section{Experimental Support}

There is no experimental data attesting to a transition of a hydrogen atom from an existing energy level $E_{n}$ to $E_{0}$. Therefore, this paper predicts that the $n=0$ state is not an energy level of the hydrogen atom, but an energy level of the electron. (In general, the expression "energy level of the hydrogen atom" is used too easily. There is a need to clearly distinguish between energy levels of the hydrogen atom and energy levels of the electron.)

Now, let us consider the case where a $\gamma$-ray is incident on the atomic nucleus (proton). 
If it is assumed that the energy of an incident $\gamma$-ray is $1.022 \mathrm{MeV}$ or higher $\left(2 m_{\mathrm{e}} c^{2}\right.$ or higher), i.e., if the energy corresponds to the sum of the mass of the electron and positron, then due to the effects of the Coulomb potential of atomic nucleus, the $\gamma$-ray may suddenly disappear and produce an electron-positron pair (electron-pair creation).

According to the author's ideas, both the electron and positron produced through pair creation in this situation are virtual particles with an energy of $-m_{\mathrm{e}} c^{2}(-0.511 \mathrm{MeV})$ prior to their creation (if the energy of each virtual particle is described using $E_{\mathrm{re}}$, then $E_{\mathrm{re}}=0$ ). Also, the electron and positron pair, which absorbed all of the energy of the $\gamma$-ray, are produced, classically speaking, near $r_{\mathrm{e}} / 2$ from the center of the atomic nucleus.

As indicated above, the electron cannot exist alone in the $n=0$ state. Therefore, $E_{0}$ cannot be regarded as an energy level of the hydrogen atom.

Next, let us find the momentum $\boldsymbol{p}_{0}$ of the electron in the $n=0$ state.

To do that, we can set $E_{\mathrm{re}}=0$ in Equation (13),

$$
\boldsymbol{p}_{0}= \pm m_{\mathrm{e}} c
$$

No problems arise if this equation is interpreted as indicating that an electron and positron forming a pair have momentums with the same magnitude but opposite directions. In addition, from Equation (47) it can be predicted that an electron with zero energy forms a pair with its antiparticle (i.e., a positron).

\section{Conclusion}

In this paper, the following equation has been derived, based on classical considerations, as the equation for the energy levels of the hydrogen atom including only the quantum number $n$.

$$
E_{n}=m_{\mathrm{e}} c^{2}\left[\left(\frac{n^{2}}{n^{2}+\alpha^{2}}\right)^{1 / 2}-1\right], \quad n=0,1,2, \cdots .
$$

However, it was determined that the $n=0$ energy level is an energy level of the electron but not an energy level of the hydrogen atom. An electron cannot exist alone in the $n=0$ state. Therefore, it is appropriate to describe the energy levels of the electron in the hydrogen atom as follows.

$$
E_{n}=m_{\mathrm{e}} c^{2}\left[\left(\frac{n^{2}}{n^{2}+\alpha^{2}}\right)^{1 / 2}-1\right], \quad n=1,2, \cdots .
$$

Incidentally, the importance of Equation (48) is not just that it predicts the existence of an $n=0$ energy level. It is already known that the energy of the electron pairs comprising the vacuum is zero. The importance of Equation (48) is that it incorporates the $n=0$ state into the equation for the energy of the hydrogen atom. In terms of profundity, Equation (48) does not match up to the Dirac equation (10) which includes multiple quantum numbers.

However, the conclusion of this paper is that Equation (48) has higher precision than the Dirac equation (12) as an equation including only the quantum number $n$.

\section{Acknowledgements}

I would like to express my thanks to the staff at ACN Translation Services for their translation assistance.

\section{References}

Suto, K. (2009). True nature of potential energy of a hydrogen atom. Physics Essays, 22(2), 135-139. http://dx.doi.org/10.4006/1.3092779

Suto K. (2011). An energy-momentum relationship for a bound electron inside a hydrogen atom. Physics Essays, 24(2), 301-307. http://dx.doi.org/10.4006/1.3583810

Schiff, L. I. (1968). Quantum Mechanics (p. 472). New York: McGraw-Hill.

\section{Copyrights}

Copyright for this article is retained by the author(s), with first publication rights granted to the journal.

This is an open-access article distributed under the terms and conditions of the Creative Commons Attribution license (http://creativecommons.org/licenses/by/3.0/). 